\title{
Current Prevalence of Hyperinsulinemia and Impaired Glucose Tolerance among PCOS Women of Telangana Region-South India
}

\author{
Roya Rozati ${ }^{*}$, Humaira Minhaj ${ }^{2}$, Wajeeda Tabassum ${ }^{3}$, Avinash Bardia4, Gautam Mehdi Ayapati ${ }^{5}$, Vikram Aiman Ayapati ${ }^{5}$, Avvari \\ Bhaskara Balaji ${ }^{6}$, Taalia Nazeer Ahmed ${ }^{7}$, and Mohammed Akbar Ali ${ }^{8}$
}

${ }^{1}$ Professor and Head, Dept of Obst \& Gynecology, Shadan Institute of Medical Sciences, Member Secretary, Maternal Health and Research Trust, Hyderabad, India ${ }^{2}$ Doctor of Pharmacy, Research Scholar, Maternal Health and Research Trust, Hyderabad, India

${ }^{3} \mathrm{MSc}$ Research Scholar, Maternal Health and Research Trust, Hyderabad, India

${ }^{4} \mathrm{PhD}$ Scientist, Maternal Health and Research Trust, Hyderabad, India

${ }^{5}$ Maternal Health and Research Trust, Hyderabad, India

${ }^{6} \mathrm{PhD}$, Lab In-charge,, IVF, Maternal Health and Research Trust, Hyderabad, India

${ }^{7} \mathrm{M}$. Pharm, Research Scholar, Maternal Health and Research Trust, Hyderabad, India

${ }^{8}$ General Medicine, Assistant Professor in the Department of Medicine, Bhaskara General Hospital and Medical College, Hyderabad, India

*Corresponding authors: Roya Rozati, Professor and Head, Dept of Obst \& Gynecology, Shadan Institute of Medical Sciences, Member Secretary, Maternal Health and Research Trust, Hyderabad, India, 500034, Phone: 9849161421; Email: drroyarozati@gmail.com

Received: 12 Mar, 2021 | Accepted: 22 Mar, 2021 | Published: 30 Apr, 2021

Citation: Rozati R, Minhaj H, Tabassum W, Bardia A, Ayapati GM, et al. (2021) Current Prevalence of Hyperinsulinemia and Impaired Glucose Tolerance among PCOS Women of Telangana Region-South India. Gynecol Women's Health Res 3(1): dx.doi.org/10.16966/2689-3096.118

Copyright: (C) 2021 Rozati R, et al. This is an open-access article distributed under the terms of the Creative Commons Attribution License, which permits unrestricted use, distribution, and reproduction in any medium, provided the original author and source are credited.

\section{Abstract}

Introduction: Polycystic ovary syndrome is the most usual endocrine disorder characterized by chronic anovulation and androgen excess. Insulin resistance, hyperinsulinemia, impaired glucose tolerance are often accompanied by PCOS and therefore must be studied to find out their prevalence and association in this condition.

Objectives: Our study is objected towards finding out the association of elevated insulin and impaired glucose tolerance with PCOS and to study the current scenario among the population of Telangana, situated in the southern region of India.

Material and Methods: We conducted a community-based study covering urban and rural areas of Telangana state. A PCOS-based questionnaire was used to recruit patients and was enrolled in the study. Probable cases and healthy controls were further evaluated for clinical and biochemical parameters. Insulin, blood glucose, HOMA-IR, ultrasonography were assessed for outcomes apart from anthropometric data.

Results: Significant results were observed for fasting glucose, fasting insulin, presence of $\geq 8$ sub capsular follicular cysts which measure $\leq 10 \mathrm{~nm}$ in sonography, HOMA-IR when probable PCOS were compared to healthy controls. Prevalence of hyperinsulinemia was $31 \%$ in probable PCOS and $8.3 \%$ among controls whereas the prevalence of impaired glucose tolerance was $35 \%$ in probable PCOS and $10 \%$ in controls.

Conclusion: Our study suggested a strong association of PCOS with hyperinsulinemia and impaired glucose tolerance in the Telangana region of South India. We thereby conclude that glucose intolerance and insulin resistance are usual among women with PCOS in our county and its association with uncontrolled diabetes mellitus in the general population might stipulate prompt and early lifestyle modifications for those who have a known family history.

Keywords: Polycystic ovary syndrome; Glucose intolerance; Insulin resistance; Hyperinsulinemia; Telangana

\section{Introduction}

Polycystic Ovary Syndrome (PCOS) is the most common condition of the endocrine which is observed in women of reproductive age group and is characterized by chronic unexplained hyperandrogenemia reporting around 95\% of hyperandrogenism in women [1]. It consists of multifarious components such as reproductive, cardiovascular, metabolic, and long- term health implications regarded as the leading cause of an ovulatory infertility with complex genetic etiology. Apart from the reproductive defects, insulin resistance and hyperinsulinemia are the hallmark features of PCOS [2]. As per the European Society for Human Reproduction and Embryology (ESHRE) and American Society for Reproductive Medicine (ASRM) appearance of any two of the three criteria as follows can be useful for diagnosing PCOS: (i) presence of polycystic ovaries on ultrasound; (ii) oligo and/or anovulation; and biochemical or clinical confirmation of hyperandrogenism on condition that other etiologies have been excluded like Cushing syndrome, congenital adrenal hyperplasia, androgen- secreting tumors, etc. Based on ultrasonography, the presence of 12 or more follicles in each ovary which measures $2-9 \mathrm{~mm}$ in diameter and increased ovarian volume $(>10 \mathrm{ml})$ is taken as the morphological diagnostic criteria [3]. One of the most notable disorders in PCOS is the manifestation of Insulin 
Resistance (IR) and associated hyperinsulinemia which is regarded as the crucial element in the pathogenesis of type 2 diabetes mellitus (T2DM) thereby having a greater risk of long term complications like metabolic disorders and numerous cardiovascular diseases [3]. The aim of our study is to establish the relationship between elevated insulin and impaired glucose tolerance in PCOS subjects among Telangana's population which is situated in the southern region of India, current scenario. This study is multicentric, and the following manuscript shows results from selected regions of Telangana as per the study protocol.

\section{Material and Methods}

A cross-sectional community-based study was conducted in Maternal Health and Research Institute, Hyderabad, Telangana, India. Ethical approval had been obtained from the institution and subjects were recruited after taking written informed consent. The main objective of the study was to find out the prevalence of patients with hyperinsulinemia or abnormal (Oral Glucose Tolerance Test) OGTT who are probable for PCOS and compare them with healthy control subjects for the same parameters. This will enable us to find the link between PCOS and hyperinsulinemia. Our inclusion criteria were women of age between 18-40 years who were dwelling in that area for more than 10 years, who were non-pregnant and non-lactating, and willing to give informed consent. Subjects who had cognitive or physical abnormalities and couldn't respond to questionnaires, subjects who had a history of drug intake which interfered with glucose metabolism were excluded from the study. Diet, lifestyle, occupation, and other environmental factors of both cases and controls were the same. A total of 60 PCOS cases and an equal integer of matched control healthy females were taken into the study. A survey of the community was done using questionnaires and subjects were selected as probable and control after various clinical, biochemical, hormonal, and ultrasonographic evaluations.

\section{Clinical and biochemical parameters}

Anthropometric data like weight, height, body mass index, waist, and the thickness of triceps and dorsum skin and presence of hirsutism, acne, acanthosis, oligomenorrhea, primary infertility was obtained from all the subjects. $10 \mathrm{ml}$ of venous blood was drawn in fasting from which $5 \mathrm{ml}$ was transferred to EDTA and the rest $5 \mathrm{ml}$ to a plain vial which was used to estimate insulin and sugar.

Insulin was measured using electrochemiluminescence immunoassay (ELICA) on Cobas e 411 analyzers and sugar was measured spectrophotometrically by making use of glucose oxidaseperoxidase method. HOMA-IR (homeostatic model assessmentinsulin resistance) for evaluating insulin resistance was calculated with the help of a formula [4].

HOMA-IR (in mass units) $=$ [fasting glucose $($ in $\mathrm{mg} / \mathrm{dl}) \times$ fasting insulin $(\mathrm{mIU} / \mathrm{L})] / 405$

Impaired Glucose Tolerance was calculated as per 1999 WHO criteria [5]

IGT=Fasting $<126$ and a $2 \mathrm{hr}$ post Glucose value in the range of 140 $200 \mathrm{mg} / \mathrm{dl}$.

\section{Ultrasonography}

In ultrasonography, the polycystic ovaries are defined by the presence of $\geq 8$ sub capsular follicular cysts which measure $\leq 10 \mathrm{~nm}$ and escalated ovarian stroma [6].

\section{Statistical analysis}

Unpaired t test was used to analyze all the continuous variables in cases and controls. Results are displayed as mean $\pm \mathrm{SD}$ and $\mathrm{P}<0.05$ is taken as statistically significant.

\section{Results}

Table 1 show the results of various clinical and biochemical parameters assessed and it is noticed that BMI, fasting blood glucose, fasting insulin and HOMA-IR, follicles on ultrasound values are significantly higher in the probable PCOS group when compared to the healthy controls. Whereas, no significant difference was observed in blood pressure. Probable PCOS subjects were selected based on Rotterdam 2003 Criteria. BMI is measured as weight in $\mathrm{kg} / \mathrm{h}$ eight in meter square. Polycystic ovaries on ultrasonography were taken for evaluation and were found to be significant for subjects with probable PCOS. Prevalence of impaired glucose tolerance was observed as 0.35 among probable PCOS and 0.1 in controls while the prevalence of hyperinsulinemia was found as 0.31 in probable PCOS cases and 0.083 among healthy controls.

\section{Discussion}

Our study demonstrates that there is a strong alliance of PCOS with insulin resistance in Telangana, South India, and is the first study in this region to evaluate these parameters and has discussed the most current scenario. We have conducted a study on the population that is relatively young suffering from PCOS and mostly having low BMI. The mean age of our subjects was $24.08 \pm 4.45$ years. The majority of such studies conducted have included subjects with a higher mean age than ours $[7,8]$. Overall abnormalities of glucose intolerance observed in our cases were $35 \%$ and $10 \%$ in controls which shows a significant difference. Insulin resistance has a very significant role in the pathophysiology of both type 2 diabetes mellitus and PCOS. Although numerous females with PCOS are at a high risk of insulin resistance, impaired glucose tolerance, and pancreatic beta- cell dysfunction [2], the exact cause of an increase in insulin levels is not yet known but could be due to elevated phosphorylation of the insulin receptors which reduces the activity of protein tyrosine kinase resulting in insulin secretion abnormality $[9,10]$. Hyperinsulinemia seems to be a crucial factor in maintaining hyperandrogenemia which acts directly to promote androgen production, elevating the effect of augmented LH stimulus which can be observed in maximum patients of PCOS [11].

Table 1: Clinical and biochemical parameters in PCOS subjects versus healthy control subjects.

\begin{tabular}{|l|c|c|c|}
\hline \multicolumn{1}{|c|}{ Parameters } & $\begin{array}{c}\text { Probable PCOS } \\
\text { Cases }(\mathbf{n}=6 \mathbf{6})\end{array}$ & $\begin{array}{c}\text { Healthy } \\
\text { Controls }(\mathbf{n}=60)\end{array}$ & P value \\
\hline Age (years) & $24.08 \pm 4.45$ & $24.89 \pm 4.32$ & 0.313 \\
\hline BMI (kg/m²) & $24.31 \pm 4.89$ & $22.57 \pm 3.18$ & $0.022^{*}$ \\
\hline Systolic Blood Pressure & $112.81 \pm 11.01$ & $113.89 \pm 8.87$ & 0.555 \\
\hline Diastolic Blood Pressure & $76.12 \pm 8.69$ & $76.15 \pm 7.75$ & 0.984 \\
\hline Fasting glucose (mg/dl) & $87.51 \pm 7.73$ & $81.79 \pm 8.65$ & $0.0002^{*}$ \\
\hline $\begin{array}{l}\text { 2 hour Post Glucose } \\
\text { (mg/dl) }\end{array}$ & $128.69 \pm 23.23$ & $118.76 \pm 21.45$ & $0.01^{*}$ \\
\hline Fasting Insulin (mIU/L) & $16.20 \pm 8.63$ & $12.93 \pm 7.26$ & $0.026^{*}$ \\
\hline HOMA-IR & $3.46 \pm 1.83$ & $2.68 \pm 1.54$ & $0.012^{*}$ \\
\hline $\begin{array}{l}\text { Ultrasonography- } \\
\text { Polycystic ovaries }\end{array}$ & $12.5 \pm 3.25$ & $6.0 \pm 2.33$ & $<0.0001^{*}$ \\
\hline
\end{tabular}




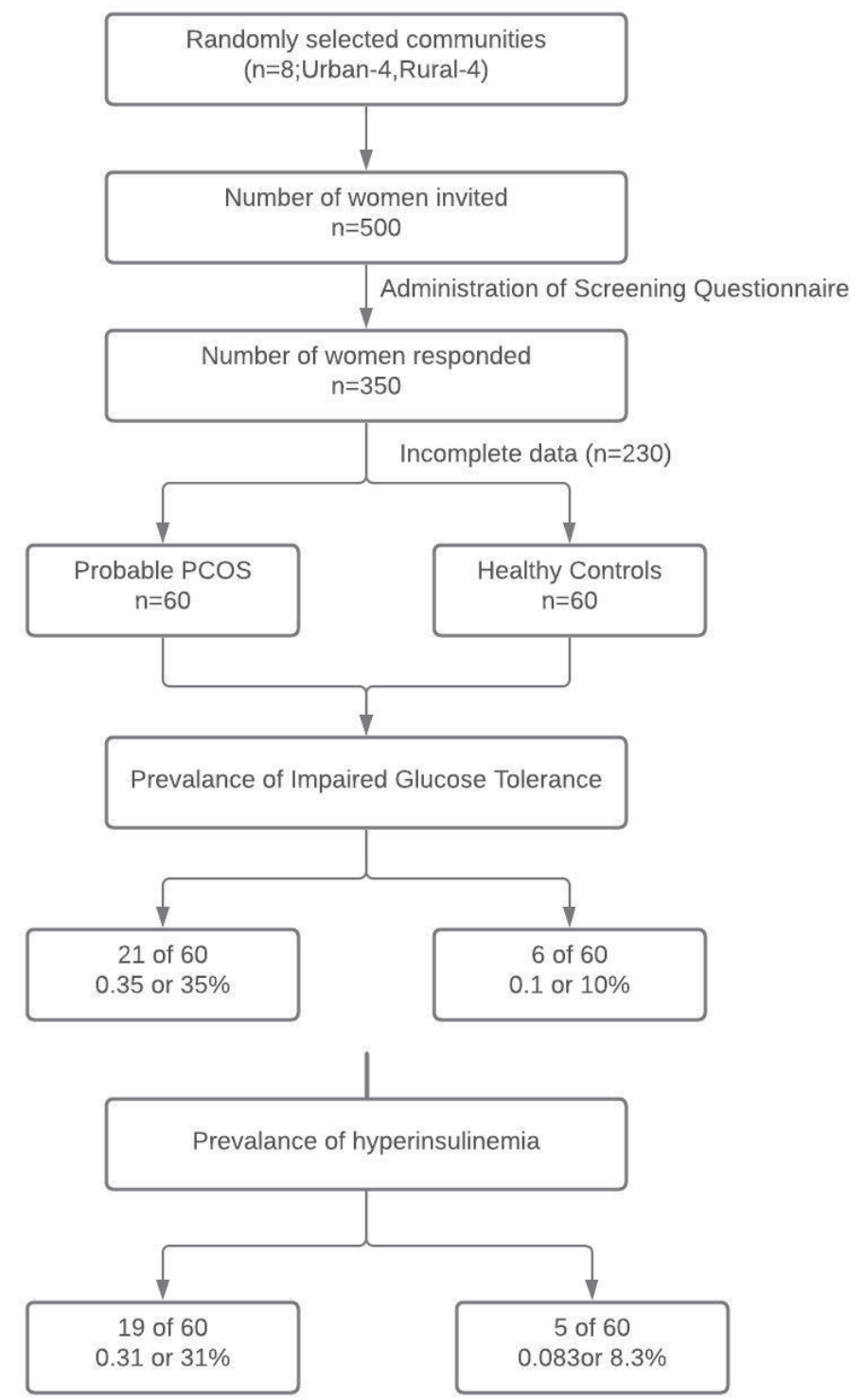

Figure 1: Flow chart showing presence of impaired glucose tolerance and hyperinsulinemia.

In our study, the prevalence of cases with hyperinsulinemia is 0.31 and 0.083 for controls (Figure 1). The probability of glucose intolerance in PCOS patients seems to be elevated equally in mixed ethnicities of the United States and Asian populations [12-14]. The compensatory mechanism of insulin in the body during hyperinsulinemia changes the steroid hormone metabolism which leads to high production of ovarian androgens, resulting in manifestations of PCOS [2]. A strong link between irregularities in the menstrual cycle and insulin resistance in PCOS women was demonstrated by studies where an ovulatory women of PCOS had shown insulin resistance and those with a regular cycle of menstruation haven't demonstrated resistance in insulin $[9,10,15]$. Insulin resistance in our study was assessed by calculating the values of HOMA-IR. Results were significantly high concerning glucose, insulin, HOMA-IR in the PCOS group when compared to control and, BMI was also observed more in cases. Insulin resistance is the cause of the excessive accumulation of fat associated with type $2 \mathrm{DM}$ and is the etiology of hyperinsulinemia [16]. Rarely, any literature is available in India on PCOS women regarding insulin resistance like the one in Delhi slums which showed a greater risk of severe insulin resistance (22\%) using HOMA-IR criteria for calculating insulin resistance [17]. From our data, we observed that $75 \%$ of women with probable PCOS who had glucose intolerance were having a family history of known DM when compared with controls that had $12 \%$ of family history of known DM. Therefore, early modification in lifestyle for such subjects can delay the onset of the disease. We also evaluated the ultrasonography and found that Glucose intolerance among PCOS patients is observed mostly in the $3^{\text {rd }}$ and $4^{\text {th }}$ decade of life [3]. 2 hours post glucose to calculate impaired glucose tolerance had been a bit challenging. Currently, in our Indian population, we observe the high prevalence of PCOS with the presence of glucose intolerance, hyperinsulinemia, family history of DM and the array starts at an early age. 


\section{Conclusion}

Women suffering from PCOS have innumerable risk factors like obesity; elevated levels of glucose, hyperinsulinemia, family history of type 2DM, menstrual irregularities, elevated cardiovascular risk factors like dyslipidemia and hypertension. Our study suggested a strong association of PCOS with hyperinsulinemia and impaired glucose tolerance in the Telangana region of South India. We thereby conclude that glucose intolerance and insulin resistance are usual among women with PCOS in our county and its association with uncontrolled diabetes mellitus in the general population might stipulate prompt and early lifestyle modifications for those who have a known family history.

\section{Acknowledgement}

We would like to express our gratitude to all the staff members of Maternal Health and Research Institute for the completion of study and we express our sincere thanks to Indian Council Of Medical Research for funding and providing us assistance in this project titled "Evaluation of prevalence, regional phenotypic variation, comorbidities, risk factors and the variations in response to different therapeutic modalities among Indian women with polycystic ovary syndrome (PCOS): A Multicenter study across India."

\section{Conflict of Interest}

No conflicts of interest.

\section{References}

1. Potau N (2004) Insulin Resistance and Polycystic Ovary Syndrome. Kumar S, O'Rahilly S (eds) Insulin Resistance: Insulin Action and its Disturbances in Disease.

2. Reddy BM, Kommoju UJ, Dasgupta S, Rayabarapu P (2016) Association of Type 2 diabetes Mellitus Genes in Polycystic Ovary Syndrome Aetiology among Women from Southern India. Indian J Med Res 144: 400-408.

3. Agarwal N, Gangopadhyay S, Koch N, Gupta A, Batra A, et al. (2015) Polycystic Ovarian Syndrome and Insulin Resistance: a North Indian Study. IJRMS.

4. Matthews DR, Hosker JP, Rudenski AS, Naylor BA, Treacher DF, et al. (1985) Homeostasis Model Assessment: Insulin Resistance and Beta-Cell Function from Fasting Plasma Glucose and Insulin Concentrations in Man. Diabetologia 28: 412-419.

5. Ashraf GM, Khurana ML, Eunice M, Gupta N, Diwivedi SN, et al. (2004) Prevalence of Glucose Intolerance Among Adolescent and Young Women with Polycystic Ovary Syndrome in India. Ind J Endocrinol Metab 6: 9.
6. Dunaif A (1997) Insulin resistance and the polycystic Ovary Syndrome: Mechanism and Implications for Pathogenesis. Endocr Rev 18: 774-800.

7. Adams J, Polson DW, Franks S (1986) Prevalence of Polycystic Ovaries in Women with Anovulation and Idiopathic Hirsutism. Br Med J (Clin Res Ed) 293: 355-359.

8. Conway GS, Honour JW, Jacobs HS (1989) Heterogeneity of the Polycystic Ovary Syndrome: Clinical, Endocrine and Ultrasound Features in 556 Patients. Clinical endocrinology 30: 459-470.

9. Dunaif A, Segal KR, Futterweit W, Dobrjansky A (1989) Profound Peripheral Insulin Resistance, Independent of Obesity, in Polycystic Ovary Syndrome. Diabetes 38: 1165-1174.

10. Robinson S, Kiddy D, Gelding SV, Willis D, Niththyananthan R, et al. (1993) The Relationship of Insulin Insensitivity to Menstrual Pattern in Women with Hyperandrogenism and Polycystic Ovaries. Clin Endocrinol 39: 351-355.

11. Marshall JC, Dunaif A (2012) All Women with PCOS should be Treated for Insulin Resistance. Fertil Steril 97: 18-22.

12. Ehrmann DA, Barnes RB, Rosenfield RL, Cavaghan MK, Imperial J (1999) Prevalence of Impaired Glucose Tolerance and Diabetes in Women with Polycystic Ovary Syndrome. Diabetes Care 22: 141146.

13. Legro RS, Kunselman AR, Dodson WC, Dunaif A (1999) Prevalence and Predictors of Risk for Type 2 Diabetes Mellitus and Impaired Glucose Tolerance in Polycystic Ovary Syndrome: a Prospective, Controlled Study in 254 Affected Women. J Clin Endocrinol Metab 84: 165-169.

14. Weerakiet S, Srisombut C, Bunnag P, Sangtong S, Chuangsoongnoen $\mathrm{N}$, et al. (2001) Prevalence of Type 2 Diabetes Mellitus and Impaired Glucose Tolerance in Asian Women with Polycystic Ovary Syndrome. Int J Gynaecol Obstet 75: 177-184.

15. Abbott DH, Dumesic DA, Franks S (2002) Developmental Origin of Polycystic Ovary Syndrome - a Hypothesis. J Endocrinol 174: 1-5.

16. Malone JI, Hansen BC (2019) Does Obesity Cause Type 2 Diabetes Mellitus (T2DM)? or is it the Opposite? Pediatr Diabetes 20: 5-9.

17. Misra A, Chaudhary D, Vikram NK, Mittal V, Devi JR, et al. (2002) Insulin Resistance and Clustering of Atherogenic Risk Factors in Women Belonging to Low Socio-economic Strata in Urban Slums of North India. Diabetes Res Clin Pract 56: 73-75. 